\title{
Region of Interest Extraction of Medical Image based on Improved Region Growing Algorithm
}

\author{
Shanshan Sun ${ }^{\mathrm{a}}$, Runtong Zhang ${ }^{\mathrm{b},{ }^{*}}$ \\ School of Economics and Management, Beijing Jiaotong University, Beijing 100044, China; \\ a15120634@bjtu.edu.cn.com, ${ }^{\mathrm{b} C}$ Corresponding author Email: 1018806359@ qq.com
}

Keywords: Medical Image, region of interest, iteration algorithm, region growing algorithm.

\begin{abstract}
Medical images are usually made up of regions of interest (ROI) and background areas, relative to the background area, the ROI contains important diagnostic information. Although the ROI may not be large in the entire image area, it is of great significance for doctors' diagnosis, clinical treatment and pathological analysis. The purpose of the paper is to extract the ROI from the surrounding environment by extracting the features of the Medical image. The paper is aimed at splitting the medical image by combining iteration algorithm and region growing algorithm, thus can mark out the nidus clearly.
\end{abstract}

\section{Introduction}

With the rapid development of Internet technology and the extensive research of image compression methods, telemedicine diagnosis, telemedicine education and remote surgery, etc. The patient can accept the consultation and treatment from the far experts in situ which save a lot of time and money for doctors and patients, and improve efficiency too. Because of the need for medical imaging diagnosis, it often requires higher accurate. Therefore, the compression of it is the lossless compression of medical image transmission. But the amount of data is often much larger than the ordinary image. So medical image lossless compression is difficult to be applied to the actual compression for its low efficiency. For medical imaging, diagnostician are interested in medical imaging and lesion area, but non lesion area occupies a large amount of information which diagnostician are not interested in. If we can reduce the information redundancy of the amount of non-lesion area, it will reduce the efficient bandwidth occupied by the medical image transmission [1, 2]. Therefore, it is necessary to segment the medical image and extract the region of interest in order to not only lose the important image information, but also reduce the storage space of the image effectively.

For medical images, the area of interest to the doctor is the lesion area, because the lesion area contains the main disease information, which is the main basis for the doctor to diagnose and formulate the treatment plan. The region of interest contains the main information in the image, so the accurate extraction of the region of interest is very important, and it is also the key problem and difficulty in the medical image pattern recognition technology. The extraction of regions of interest is of great importance for medical image processing. Medical image is rich in content and has complex structures. How to extract information which contains diseases (such as cancer, calcification and inflammation) region is the main problems and difficulties for the medical image retrieval and recognition, and it is very important for doctors to diagnose the disease, clinical treatment, teaching and scientific research and case analysis. The accurate extraction of ROI not only can improve the retrieval efficiency, but also better classify and classify the pathological signs. Through in-depth study of biology, cognitive psychology and computer science for many years and the accumulation of interdisciplinary research, researchers gradually reach a consensus that ROI can replace the original image in image processing for the reason the image contained in the ROI images are the main information and key information [3]. When dealing with images, we focus on the analysis of ROI in the image, which can reduce the amount of calculation, and better deal with the rapid growth of image data. 
At present, the commonly used methods of medical image segmentation include several kinds [3] :(1) Pixel based methods. This method only considers the pixels in the image itself, but does not take advantage of other information in the image, such as spatial location information, texture information and so on, so this method is generally used for preprocessing images. (2) Region based approach. In addition to using the pixel itself, this method also takes into account the spatial relationship between pixels, and the segmentation results are connected, and this is a local segmentation method. (3) Boundary based approach. This method can be said to be the earliest research method, mainly to solve the problem of image segmentation by using the characteristics of the edge gray value change which is often more intense. (4) Model-based approach. This method is a hot issue in this field. It mainly uses artificial participation or prior knowledge to guide image segmentation. (5) Combination of algorithms. The advantages of various algorithms are combined to achieve accurate segmentation of images.

There are automatic and nonautomatic methods for the extraction of regions of interest. Automatic extraction generally is based on the image segmentation idea, but there is not an ideal segmentation method can completely segmented semantic block. Non automatic extraction is entirely up to the user to draw the region of interest, but human intervention and the workload is so large that it is not conducive to intelligent image retrieval system for medical chart. To solve this problem, this paper combines the region growing method with threshold segmentation, and proposes an improved region growing method, and realizes the image segmentation and extraction using the algorithm on the Matlab platform.

\section{Literature Review}

In recent years, researchers have made many achievements in the image segmentation, which is more close to and patronize the people's livelihood in the field of medicine.

At present, some people have achieved good segmentation results by means of improving the region growth method. For example, Hu Xiaodan have researched the method of segmenting the image fast based on the Gaussion statistical model[4], who has established Gaussion statistical model based avail oneself of the noise, which adopts the method of maximum minimum to improve the segmentation method and get better segmentation results. Wang Xueong and other people have put forward a method based on HSI weights in color image segmentation [5], which makes the color natural disaster image has been effectively partitioned. Wangnan et al are committed to the study of color image segmentation [6], and combine with color information and get a good segmentation based on the gray image processing. Thomas LORENZ has improved the regional growth method [7], which expanded the use of regional growth laws.

At the same time, researchers have done a lot to find the optimal threshold. For example, wei Hongli et al. have constructed an orthogonal multiple wavelet based on the research of medical image threshold segmentation algorithm, which expands the image in a scale space and use different thresholds to process segmentation for the wavelet coefficients at different resolutions. The experimental results show that the method can obtain a clear target area and edge characteristics. Ren Mingwu et al. have raised a new method of constructing histogram [9], which increased the resistance of the threshold based on the edge pattern. Song Yudan and others have used a combination of the minimum threshold method and the Laplace's histogram to determine the threshold, the algorithm has obtained the ideal segmentation effect and has made some breakthrough in the selection of color image threshold.

In addition, $\mathrm{Fu}$ Zengliang et al have used a combination of the traditional method and the horizontal set method for image segmentation [11]. A desired result was obtained. Zhu Lingli and others combined genetic algorithm and cluster analysis to study CT image segmentation and obtained good application [12]. Liu and others have used a combination of the method of regional growth and the texture information to split image and got a good effect [13]. Wang and others use the CV model based on a priori shape to split the liver CT image [14], which achieved a good anti-interference effect. Liu Ziqi and others have proposed a multi-regional method to split the liver CT image, which 
can complete the segmentation quickly and accurately [15]. Liu Changzheng et al propose an improved level set based CT liver image segmentation based on the non-parametric probability model [16], the method has the characteristics of high precision, strong noise immunity and so on. Qiu Ming and others use the improved GVF model for CT image segmentation [17], which get a better effect than the original model.

\section{Improved regional growth algorithm}

\subsection{Regional Growth Algorithm.}

The traditional regional growth algorithm starts at a certain pixel, and assembles the pixels that have the similarity into a region according to certain guidelines. The algorithm first selects the initial pixels in the area where the segmentation is needed, the initial pixels is also called seeds. Then the seed incorporates the pixel that has the same or similar value in the surrounding area into the area of the seed pixel according to certain "growth rule", and these new pixels are then taken as new seed pixels and continue with the process until they no longer have a pixel that conforms to the growth rule. The rules of growth have a large effect on the segmentation of images.

\subsection{The Description of Improved Regional Growth Algorithm.}

We can divide the image with the traditional regional growth method, but if the area we select is too small, the target area cannot be fully separated; on the contrary, if the region we pick is too large, although we can fully separate the target area, we will also separate the areas that are not necessary, which can lead to the over segmentation. Because the traditional regional segmentation algorithm cannot obtain satisfactory results, in this paper, we want to make full use of the complementarity of multiple segmentation methods, and use the iterative method to improve the traditional regional growth algorithm in order to achieve the purpose of improving segmentation.

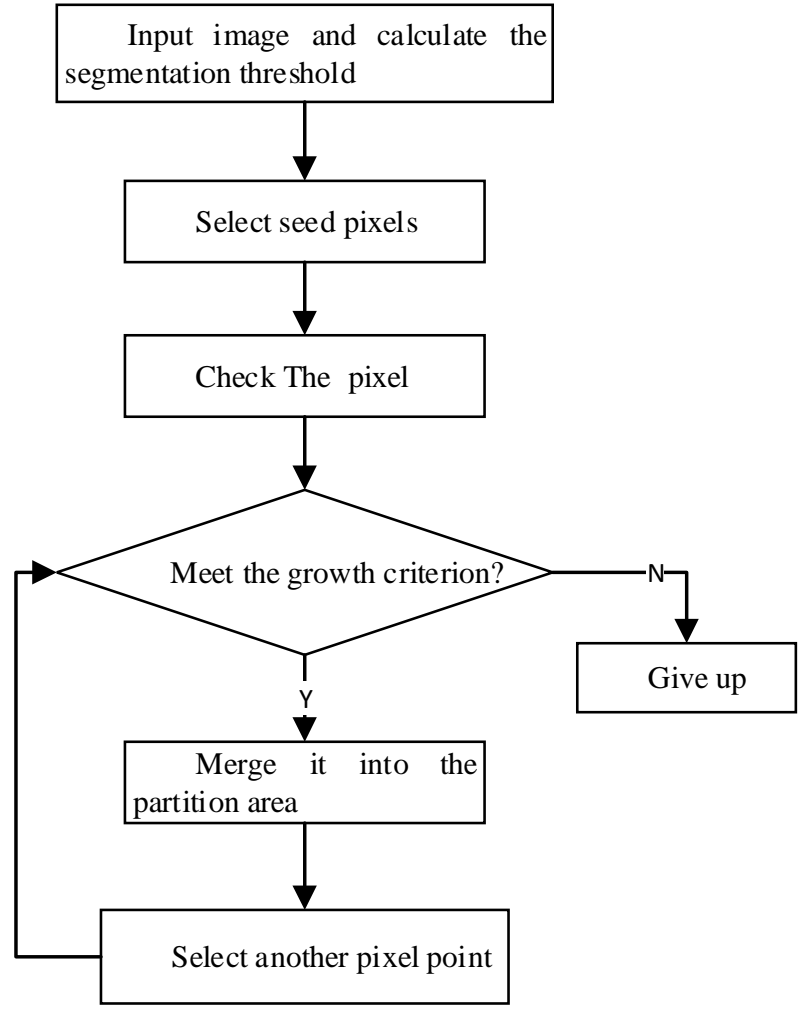

Fig. 1 The flow chart of regional growth

This paper puts forward the method of segmentation based on iterative method and regional growth method. First, the threshold segmentation method is used to calculate the optimal segmentation threshold, and then the threshold is used as the criterion of regional growth criterion. This article mainly extends the growth sentence condition of regional growth. Other studies have generally used the grey gradient of the adjacent pixel and the seed pixel to be a growth sentence 
condition, but here, the paper used a location-based grey scale as a growth sentence, the following is the consistency standard and the rules for its birth.

Growth rule: If the gray difference between the pixel to be detected and the seed pixel point is smaller than the growth threshold $\mathrm{T}$, then we can think the pixels are similar to the seed pixels, and add it to the partitioned target area. On the contrary, if the gray difference between the pixel to be detected and the seed pixel point is bigger than the growth threshold $\mathrm{T}$, then we can think that the pixels are not the target area.

The algorithm description is shown in figure 1:

(1) Input the image and compute the optimal segmentation threshold T;

(2) Select the seed pixels;

(3) Check a pixel point, calculate the grey value between the pixels and the pixels of the seed, if the grey value is less than the threshold $T$, which means the point meets the growth criteria, and then add it to the target area. On the contrary, if the grey value between the two is greater than the threshold $\mathrm{T}$, which means the point not meets the growth criteria and give up that point;

(4) Repeat steps (3) until the pixels in the image are checked.

\section{Results and Analysis}

In this paper, the algorithm is programmed by Matlab, and the method is used to segment liver images automatically.

This article is a semi-automatic image segmentation, and the first is to select the seed pixels, as shown in figure 2, we can select multiple or single seed pixels; in the paper, we take a pixel for example to segment the image, the highlights in figure 2 represent the selected seed points, and the figure 3 is the result of the split. From figure 3, we can see that the algorithm has separated the points which are similar to the pixels of the seed pixels, which demonstrates its effectiveness against image segmentation.

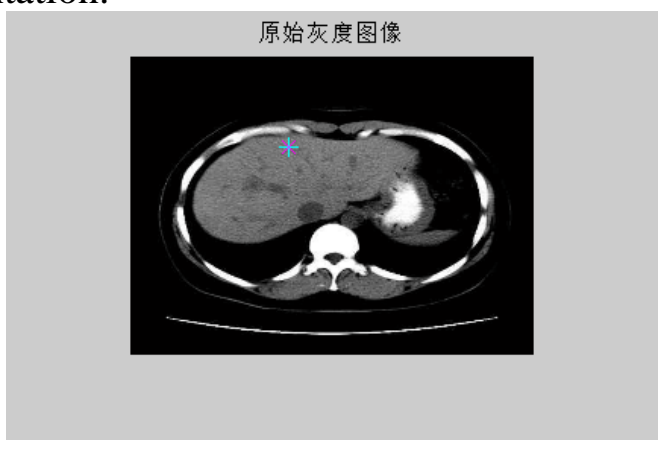

Fig. 2 The original image

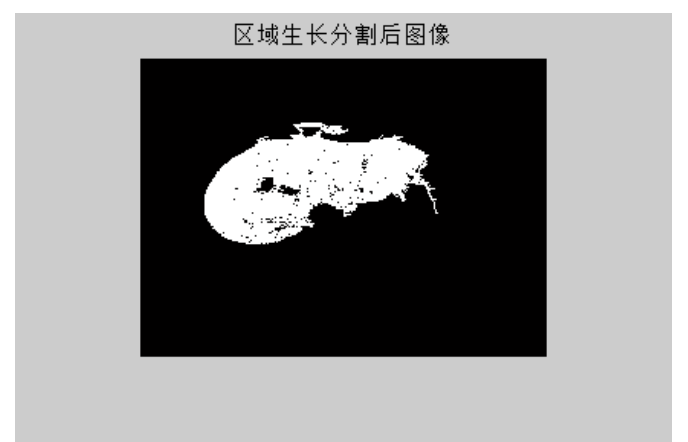

Fig. 3 The segmented image

\section{Summary}

The segmentation of medical imaging is a common problem in the medical field. This paper designs and implements the method of segmentation based on threshold method and regional growth method after analyzing the characteristics of threshold method and regional growth method, and then implements the image segmentation on the Matlab platform.

\section{Acknowledgments}

This work was partially supported by a Key Project of National Natural Science Foundation of China (NSFC) with grant number 71532002.

\section{References}

[1]. Xie Wei-ying. Study on the detection in medical lesions [D]. Lanzhou University, 2014 
[2]. Liu Hang, Wang Dong, Pei Xi, Cao Rui-fen, HU Li-qin, et al. Liver Segmentation Research Using Improved Region Growing Algorithm [J]. Chinese Journal of Medical Physics, 2014, 31(5):5204-5208

[3]. Kutter M, Bhattacharjee S K, Ebrhimi T.Towards second generation watermarking schemes[C]//IEEE Int Conf on Image Processing(ICIP 99),Kobe,Japan,1999.

[4]. Hu Xiao-dan, LI Wen, Liu Hai-bo. A Fast Image Region Segmentation Method Based on Gaussion Statistical Model [J]. Journal of Fujian Normal University (Natural Science Edition), 2011, 27(2):133-137.

[5]. Wang Xuedong, Zhou Mingquan, Fan Yachun, Li Peng. The algorithm of graph cut using HSI weights in color image segmentation [J]. Journal of Image and Graphics, 2011, 16(2):221-226.

[6]. Wang Nan, Huang Yang-cheng. A Method of the Region Segmentation and Edge Pick-Up in Color Image [J]. Journal of Institute of Command and Technology, 1999, 10(4):106-110.

[7]. Lorenz T. Set-valued maps for image segmentation [J]. Computing and Visualization in Science, 2001, 4(1):41-57.

[8]. Wei Hong-li, Yu Xin-bo, Zhao Wen-cang, Gao Yan-chen. The Medical Image Threshold Value Segmentation Algorithm Study Based on Multi-Wavelet Analysis [J]. Computerized Tomography Theory and Applications, 2009, 18(1):8-15.

[9]. Ren Ming-wu, Yang Jing-Ning, Sun Nan. NEW HISTOGRAM MODIFICATION BASED ON IMAGE EDGE MODEL [J]. Journal of Computer Research and Development, 2001, 38(8):972-976.

[10]. Wu Qing, Chen Sai-ming, Tu Rong. Application of FCM and Level Set in Medical Image Segmentation [J]. China Medical Devices, 2012, 27(9):38-41.

[11]. Fu Zeng-liang, Chen Xiao-jun, Ye Ming, Li Feng, Wang Cheng-tao. Method for Heart CT Image Segmentation [J]. Method for Heart CT Image Segmentation, 2009, 35(12):189-191.

[12]. Zhu Ling-Li, LI Ji-Gui, Bao Su-Su. Application of Clustering Analysis Based on Genetic Algorithm in CT Image Segmentation [J]. Computer Science, 2006, 33(10):186-188.

[13]. Wang Hao, Kang Xiao-dong, Liu Ling-ling, Guo Hong, Guo Jun, Geng Jia-jia. Medical image segmentation based on wavelet transform and Markov field [J]. Journal of Computer Applications, 2011, 31(s2):140-142.

[14]. Wang Xiao-fang, Zhao Yu-qian. Liver CT image segmentation based on prior shape CV model [J]. Journal of Optoelectronics Laser, 2010(6):953-956.

[15]. Liu Zi-qi, Han Xu-li, Chen Zhi-jun. A New Segmentation Method for CT Images with Multiple Liver Regions [J]. Computer Simulation, 2008, 25(10):202-205.

[16]. Liu Chang-zheng, Ma Jun-lin. Segmentation of Liver CT Image Based on Nonparametric Density Estimates [J]. Journal of Harbin University of Science and Technology, 2008, 13(4):62-65.

[17]. Qiu Ming, Zhang Er-hu, Zhang Zhi-gang. Method of CT Image Segmentation Based on Modified GVF Model [J]. Mini-micro Systems, 2006, 27(1):155-157. 